\title{
What is the impact of a novel MED12 variant on syndromic conotruncal heart defects? Analysis of case report on two male sibs
}

Silvia Amodeo ${ }^{1}$, Giuseppe Vitrano ${ }^{1}$, Melania Guardino ${ }^{2 *}$, Giuseppe Paci ${ }^{1}$, Fulvio Corselli', Vincenzo Antona ${ }^{2}$, Giuseppe Barrano ${ }^{3}$, Monia Magliozzi ${ }^{4}$, Antonio Novelli ${ }^{4}$, Renato Venezia ${ }^{1}$ and Giovanni Corsello ${ }^{2}$

\begin{abstract}
Background: Syndromic congenital heart disease accounts for $30 \%$ of cases and can be determined by genetic, environmental or multifactorial causes. In many cases the etiology remains uncertain. Many known genes are responsible for specific morphopathogenetic mechanisms during the development of the heart whose alteration can determine specific phenotypes of cardiac malformations.
\end{abstract}

Case presentation: We report on two cases of association of conotruncal heart defect with facial dysmorphisms in sibs. In both cases the malformations' identification occurred by ultrasound in the prenatal period. It was followed by prenatal invasive diagnosis. The genetic analysis revealed no rearrangements in Array-CGH test, while gene panel sequencing identified a new hemizygous variant of uncertain significance (c.887G > A; p.Arg296GIn) in the MED12 gene, located on the $\mathrm{X}$ chromosome and inherited from the healthy mother.

Conclusion: No other reports about the involvement of MED12 gene in syndromic conotruncal heart defects are actually available from the literature and the international genomic databases. This novel variant is a likely pathogenic variant of uncertain significance and it could broaden the spectrum of genes involved in the development of congenital heart diseases and the phenotypic range of MED12-related disorders.

Keywords: Congenital heart diseases, Conotruncal heart defects, Facial dysmorphisms, MED12, Echocardiography, Next generation sequencing, Case report

\section{Background}

Conotruncal heart defects (CTDs), account for approximately $30 \%$ of congenital heart disease (CHDs). They are characterized by anomalies of the cardiac outflow tract or of the great arteries [1]. These include common arterial trunk (CAT) and aorto-pulmonary window (APW), as well as

\footnotetext{
* Correspondence: melania.guardino@hotmail.it

${ }^{2}$ Department of Neonatology and NICU, University Hospital Policlinico P. Giaccone, Palermo, Italy

Full list of author information is available at the end of the article
}

double outlet right ventricle (DORV), tetralogy of Fallot (TOF) with or without pulmonary atresia (PA), transposition of the great arteries (TGA), interrupted aortic arch (IAA).

CAT is characterized by a single great artery arising from the ventricles, giving origin to aorta, coronary arteries and pulmonary arteries, and by a ventricular septal defect (VSD) [2]. According to the Collett and Edwards' Classification [3], CAT is distinguished in: type I when a single pulmonary trunk arises from the truncus arteriosus, type II when two pulmonary branches arise separated but close from the posterior wall of the truncus 
arteriosus, type III when two pulmonary branches arise separated from lateral walls of the truncus arteriosus. Type IV now is defined pulmonary atresia with ventricular septal defect (PAVSD).

Aorto-pulmonary window (APW) is a communication between the ascending aorta and the pulmonary artery in the presence of separate semilunar valves [4]. It was classified by Richardson into three types [5]: in type I the defect between the aorta and the main pulmonary artery is immediately above the sinuses of Valsalva, in type II the defect is located more distally between the ascending aorta and the origin of the right pulmonary artery and in type III there is anomalous origin of the right pulmonary artery from the ascending aorta.

Both CAT and APW are determined by the failure of septation of the physiologic arterial common trunk into aorta and pulmonary artery trunk during development of the ventricular outlets and proximal arterial segment of the heart tube.

We report on a novel variant in the MED12 gene, detected by Next Generation Sequencing (NGS), associated with recurrent conotruncal heart defects, CAT and APW respectively, and facial dysmorphisms in an Italian family.

\section{Case presentation}

\section{Case 1}

A couple, 33 year-old woman and 34 years-old man, referred to Prenatal Diagnostic Unit of Policlinico Universitario Paolo Giaccone for a routine mid-trimester ultrasound scan of their first pregnancy. Conception was spontaneous, there was no consanguinity and the couple was in excellent health without any medical history. They had decided to not perform the first trimester screening for fetal aneuploidy.

The mid-trimester ultrasound scan, performed at 20.1 weeks of gestation, showed a male fetus with normal four-chamber view but abnormal outflow tract views: a single great artery equal overrode a ventricular septal defect (VSD) (Fig. 1a). The main pulmonary trunk and subsequent bifurcation arose from the single great artery immediately distal to the truncal valve. Color Doppler confirmed the blood flow through the VSD, the overriding artery, the origin of the pulmonary artery and its bifurcation. This finding suggested a diagnosis of truncus arteriosus type I with VSD according to the Collett and Edwards' Classification [3]. Ultrasound research of associated extra-cardiac abnormalities has highlighted microretrognathia and nuchal edema (Fig. $1 \mathrm{~b}$ and $\mathrm{c}$ ).

CAT is often associated with extra-cardiac anomalies in the context of specific syndromes, the most common is 22q11 microdeletion [2], so, following genetic counseling, amniocentesis for karyotype and Array-CGH (array comparative genomic hybridization) was performed. The Array-CGH was performed with CytoSure ISCA v2 Arrays $8 \times 60 \mathrm{k}$. Both the standard karyotype and the CGHarrays were negative $(\operatorname{arr}(1-22) \times 2,(\mathrm{XY}) \times 1)$.

The couple opted for the voluntary termination of the pregnancy and, on the advice of geneticists, requested to store fetal DNA.

\section{Case 2}

One year later, the same couple referred again to our Prenatal Diagnostic Unit for the second pregnancy at

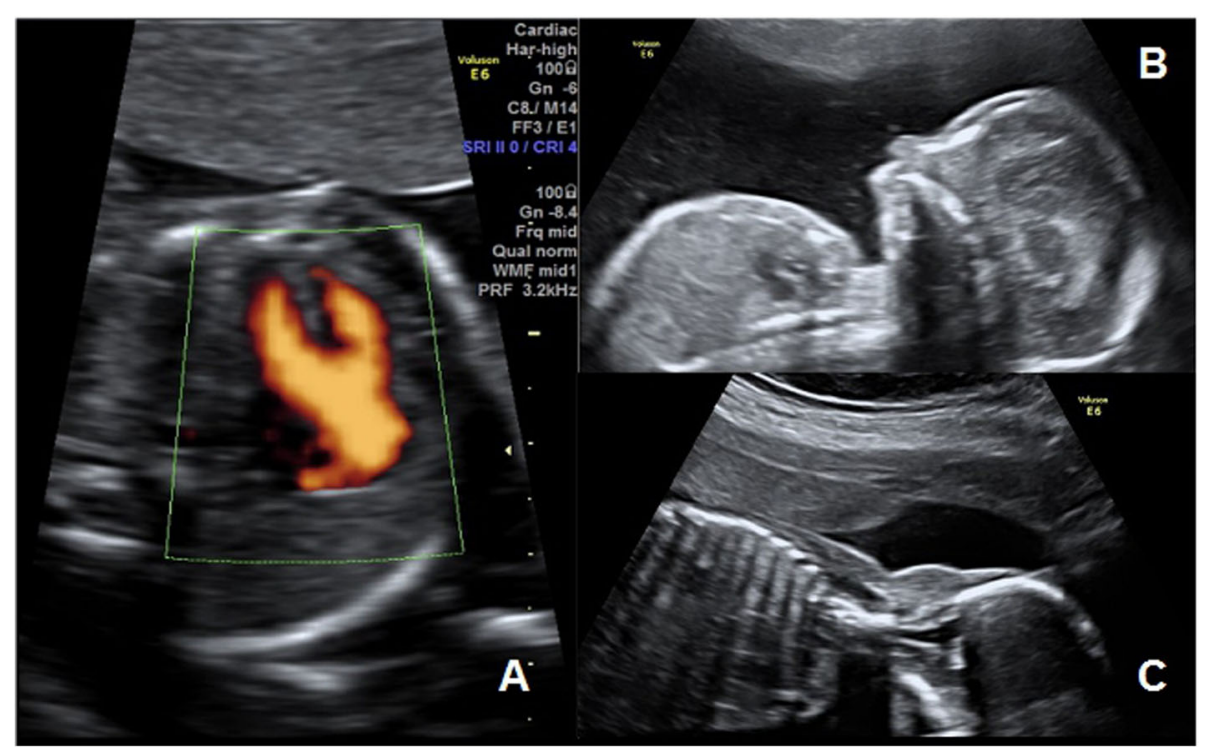

Fig. 1 Case 1 mid-trimester ultrasound scan. a single great artery that equal overrode a ventricular septal defect; b microretrognathia; c nuchal edema 
12.5 weeks of gestation for first trimester combined screening for fetal aneuploidy. Free $\beta$-hCG and PAPP-A were $1.397 \mathrm{MoM}$ and $1.55 \mathrm{MoM}$. Nuchal translucency was $1.4 \mathrm{~mm}$, nasal bone present, FHR $159 \mathrm{bpm}$, ductus venosus PI 1.15 and tricuspid regurgitation and microretrognathia were detected (Fig. 2a). Ultrasound showed no other fetal anomalies evaluable at the first trimester. Patient-specific risks for trisomy 21, 18, 13, computed based on the FMF algorithm, were 1/350, 1/1862 and < $1 / 20.000$ respectively. Considering the tricuspid regurgitation and microretrognathia and the history of fetal malformation in the previous pregnancy, following genetic counseling, the couple declined villocentesis via karyotype and microarray analysis. Therefore, early fetal echocardiography was performed at 18 weeks: three vessels and trachea view showed a communication between aorta and pulmonary artery immediately above the sinuses of Valsalva, so an aorto-pulmonary window type I according to Richardson's Classification [5] (Fig. 2b) and thymic hypoplasia. A detailed ultrasound to assess fetal anatomy confirmed the microretrognathia in male fetus. Therefore, following genetic counseling, the couple accepted amniocentesis for karyotype and Array-CGH. The Array-CGH was performed with SurePrint G3 Human CGH Microarray 8x60k. Again, both the standard karyotype and the Array-CGH were negative (arr (1-22) $\times 2,(\mathrm{XY}) \times 1)$ and the couple decided to the voluntary termination of the pregnancy and to store fetal DNA.

The autopsy of aborted fetuses confirmed in both male sibs the conotruncal heart defects, truncus arteriosus in the first fetus and aorto-pulmonary window in the second, the same facial dysmorphisms (microretrognathia, small mouth, low-set ears) and sandal gap (Fig. 3).

Considering the recurrence of the association of conotruncal hearts disease and facial anomalies in both sibs, obtained informed consent for genetic testing, molecular genetic study was performed on genomic fetal DNA extracted from amniotic fluid through Next Generation Sequencing (NGS), evaluating cardiopathy-associated genes (Table 1). We used targeted resequencing with a uniquely customized design SeqCap EZ Custom Enrichment Kit (Roche Life Science) on Illumina sequencing platform (NextSeq550, San Diego, CA). We used the BaseSpace pipeline (Illumina, https://basespace.illumina. $\mathrm{com} /$ ) and the VariantStudio software (Illumina, http:// variantstudio.software.illumina.com/) for the variant calling and annotating variants, respectively. Sequencing data were aligned to the hg19 human reference genome. We analyzed the variants in silico by using ScaleInvariant Feature Transform (SIFT), Polymorphism Phenotyping v2 (PolyPhen-2) for the prediction of deleterious non-synonymous SNVs for human diseases. The analysis revealed the hemizygous variant, NM_005120.2: c.887G > A (p.Arg296Gln) in MED12 gene located on the $\mathrm{X}$ chromosome. Sanger sequencing following a standard protocol (BigDye Terminator v3.1 Cycle Sequencing Kit, Applied Biosystems by Life Technologies) confirmed the variant and we tested it for familial segregation using the maternal DNA and the fetal DNA from the first pregnancy showing the heterozygous and hemizygous state respectively. This finding may explain the fetal phenotype and X-linked trasmission. The whole exome sequencing (WES) was not performed in relation to the diagnostic relevance of the genetic results.

\section{Discussion and conclusions}

The prenatal finding of recurrent CTDs, truncus arteriosus and aorto-pulmonary window respectively, associated with facial dysmorphisms in an Italian family gave us the suspicious of a syndromic CHDs, inducing the search for a possible genetic cause.

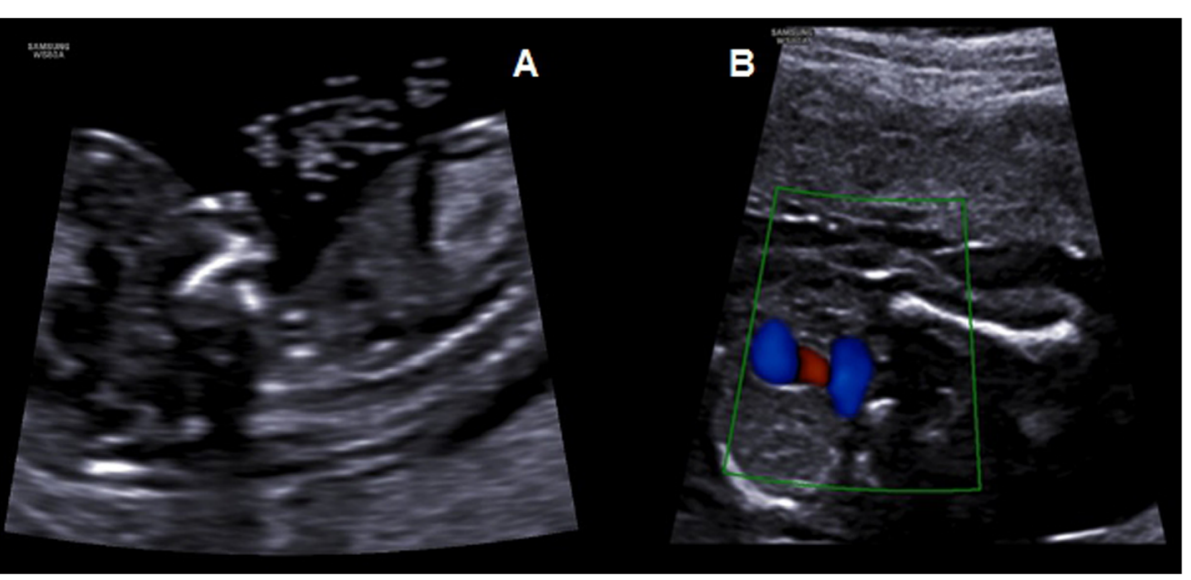

Fig. 2 Case 2 ultrasound finding. a first-trimester ultrasound scan shows microretrognathia; $\mathbf{b}$ three vessels and trachea view shows the communication between aorta and pulmonary artery (aorto-pulmonary window) 


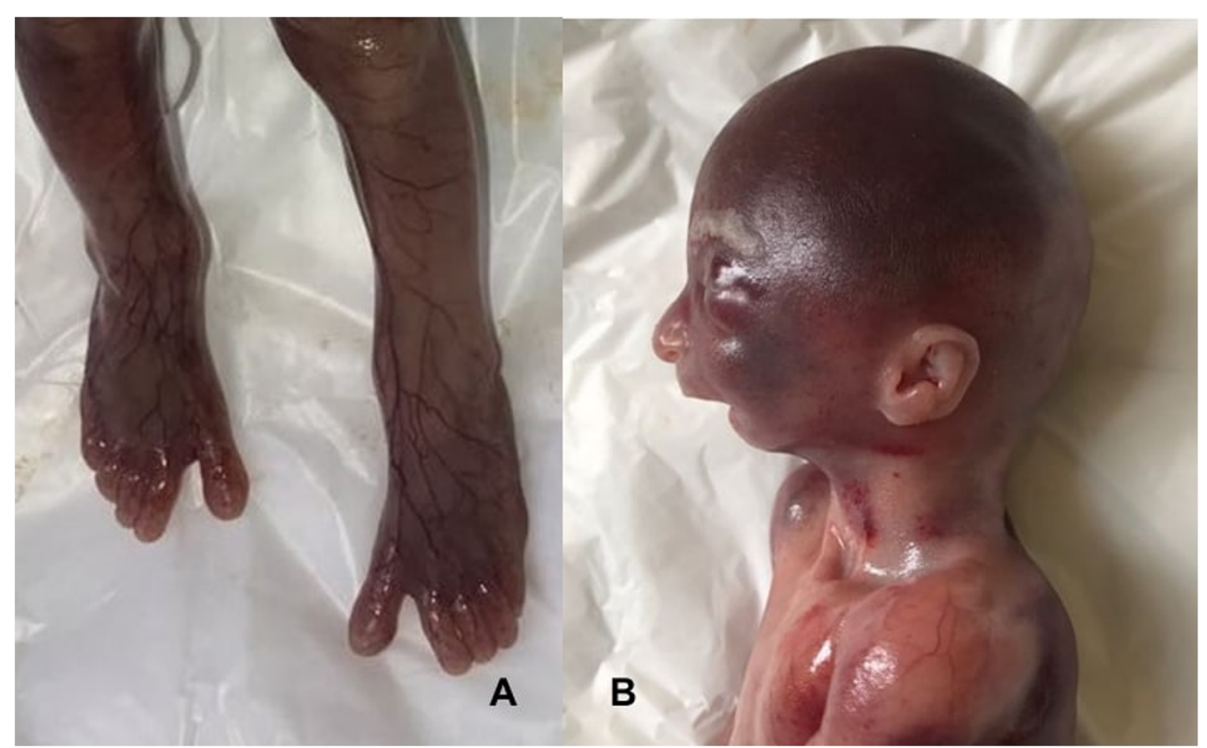

Fig. 3 Autopsy findings:: a sandal gap from first case; b microretrognathia, small mouth and low-set ears from second case

Congenital heart defects (CHDs) are structural defects due to abnormal cardiac development in embryos. Approximately $70 \%$ of CHDs present as an isolated malformation (non-syndromic CHD), while $30 \%$ are syndromic because in association with other extra-cardiac anomalies [6]. Causes of CHDs are genetic in $8 \%$, environmental in $2 \%$ and multifactorial or not determined in about $90 \%$ of cases [7]. The genetic mechanisms responsible for CHD are complex, heterogeneous and incompletely understood: approximately $8-12 \%$ is due to chromosomal anomalies, $3-25 \%$ to copy number variation and $3-5 \%$ to single gene defect [8]. These genetic variants, de novo or inherited, concern genes involved in normal cardiac development, altering signal transduction, transcriptional regulation and encoding proteins. In some cases the same genes have pleiotropic effects on other organs [8] and this explains syndromic CHDs.

Clark EB [9] classified the CHDs according to the pathogenesis: conotruncal defects are classified among the anomalies of the migration of the ectomesenchyme, in which there is an anomalous migration of component of the secondary/anterior heart field. During heart development the outflow tract develops by adding cells from the pharyngeal mesoderm called the secondary/anterior

Table 1 Cardiopathy-associated genes evalueted

\begin{tabular}{|c|c|c|c|c|c|c|}
\hline ACTA2 & CFC1 & FBN2 & GDF2 & $\mathrm{MYH11}$ & RASA1 & TGFB2 \\
\hline ARHGAP31 & CRELD1 & FBN1 & GATA4 & MYLK & RBM10 & TGFB3 \\
\hline ACVR2B & CITED2 & FMN1 & GDF1 & MYH6 & RBPJ & TGFBR1 \\
\hline ACVRL1 & COL3A1 & FMN2 & HAND2 & MED12 & SALL4 & TGFBR2 \\
\hline ADAMTSL4 & DOCK6 & FOXC1 & HAND1 & MED12L & SLC2A10 & TGFBR3 \\
\hline BMPR1B/ALK6 & DLL4 & FOXH1 & $\mathrm{HEY} 2$ & NODAL & SMAD3 & TOPBP1 \\
\hline BCOR & DIAPH2 & FHOD1 & ISL1 & NFATC1 & SOX7 & TFAP2B \\
\hline bcl9l & DNAH11 & FGF8 & LEFTY1 & NKX2-5 & SKI & TBX1 \\
\hline BMP4 & DNAH5 & FHOD3 & LEFTY2 & NAT10 & SMAD4 & TBX2 \\
\hline BMPR2 & DIAPH1 & FOXA2 & JAM3 & NKX2-6 & SMAD1 & TBX4 \\
\hline B3GAT3 & EIF2AK4 & FOXF1 & JAG1 & NOTCH1 & SMAD6 & TBX20 \\
\hline CRKL & ELN & FLNA & KCNK3 & $\mathrm{NOTCH} 2$ & SMAD9 & TBX3 \\
\hline CBLN2 & EFEMP2 & GDF1 & MCTP2 & $\mathrm{NOTCH} 3$ & SALL1 & $\mathrm{ZIC3}$ \\
\hline CBS & ENG & GJA1 & MID1 & PITX2 & TLL1 & ZFPM2/FOG2 \\
\hline CAV1 & ETS-1 & GJA5 & MAPK1 & PPARG & SHROOM3 & ZDHHC9 \\
\hline CFAP53 & EOGT & GATA6 & MHC6 & PLOD1 & TGFB1 & \\
\hline
\end{tabular}


cardiac field [10], whereas the aorto-pulmonary septum develops by condensation of cardiac neural crest (CNC) cell - derived mesenchyme [11]. Alteration or loss of these developmental processes leads to conotruncal anomalies. The increasing studies of the genetic etiology of CHDs allowed the identification of many genes involved in specific morphogenetic mechanisms and responsible of specific cardiac phenotype, creating a link between cause, pathogenetic mechanism and type of malformation [12]. Conotruncal heart diseases are associated with various chromosomal pathologies and monogenic syndromes: the most frequent associations of the CAT are with DiGeorge syndrome, interstitial duplication of $8 \mathrm{q}$ and CHARGE syndrome, whereas the APW can be associated to VACTERL association and CHARGE syndrome [13]. Among the genetic variants associated with conotruncal defects, the MED12 gene does not currently appear. MED12 gene is located on chromosome $\mathrm{X}$ and encodes a subunit of the Mediator Complex, called mediator complex subunit 12, which is crucial in RNA polymerase II transcription [14]. Zebrafish models have shown that Med12, being coregulator of specific transcription factors, is necessary during development: med12 zebrafish mutants showed defects in neural crest formation, chondrogenesis and organogenesis of the brain, liver, pancreas and kidney $[15,16]$. Zebrafish and mouse MED12-mutant demonstrated the involvement of the MED12 gene in heart development $[17,18]$.

Our study reports for the first time the finding of variant in the MED12 gene associated with syndromic CHD, specifically associated with facial dysmorphisms. A probable explanation of the role of the MED12 gene in determining conotruncal defect would be found in the molecular mechanisms involved in embryonic development of the heart: Med12 regulates gene-specific functions during development and it is necessary for correct $\mathrm{Wnt} / \beta$-catenin signaling and $\mathrm{Wnt} /$ planar cell polarity (PCP) pathway, as demonstrated on mouse models by Rocha et al. [18] The consequent alteration of the Wnt pathway and of the PCP signaling would therefore be responsible for the lack of migration of the CNC cells during the formation of the aorto-pulmonary septum. Schleiffarth et al [19] have indeed shown that the Wnt signal, in particular Wnt5a, is necessary for the correct development of the aorto-pulmonary septum and that its loss is responsible for the development of conotruncal defects.

The variant reported in our study is not described in the international literature (PubMed/Medline) and in the Catalog of Human Genes and Genetic Disorders (OMIM) and it is currently to be considered VOUS (Variants Of Uncertain Significance). The normal phenotype of the mother carrying the variant on the $\mathrm{X}$ chromosome in heterozygosity and the recurrence of the pathological phenotype in fetuses both male with hemizygous state increase the suspicion that this variant is the cause of the pathology in these family patients and that it may explain an X-linked transmission of syndromic conotruncal defects, contributing to increase the phenotypic range of MED12-related disorders. Therefore it will have important implications for genetic and preconception counseling, family planning and clinical management.

In conclusion, despite we know many genetic variations involved in the development of CHDs and specifically of CTDs, in most patients the genetic defects remain unknown [1]. The latest investigation techniques such as next generation sequencing (NGS) could increase our knowledge about CHD genetic etiology. The variant c.887G > A (p.Arg296Gln) in MED12 gene is a likely pathogenic VOUS and it could shed new light on the possible pathogenesis of conotruncal heart defects.

\section{Abbreviations \\ CTDs: ConoTruncal defects; CHDs: Congenital heart disease; CAT: Common arterial trunk; APW: Aorto-pulmonary window; DORV: Double outlet right ventricle; TOF: Tetralogy of Fallot; PA: Pulmonary atresia; TGA: Transposition great arteries; IAA: Interrupted aortic arch; VSD: Ventricular septal defect; PAVSD: Pulmonary atresia with ventricular septal defect; NGS: Next Generation Sequencing; Array-CGH: Array comparative genomic hybridization; VOUS: Variants Of Uncertain Significance}

\section{Acknowledgements}

Not applicable.

\section{Authors' contributions}

All authors have participated in the diagnostic pathways. All authors have read and approved the final manuscript.

\section{Funding}

None.

Availability of data and materials

All the data presented in this article are stored in Prenatal Diagnostic Unit of University Hospital Policlinico Paolo Giaccone.

Ethics approval and consent to participate

Not applicable.

Consent for publication

For the publication, the authors have obtained consent from the couple.

Competing interests

The authors declare that they have no competing interests.

\section{Author details}

${ }^{1}$ Department of Obstetrics and Gynecology, University Hospital Policlinico P. Giaccone, Via Alfonso Giordano 3, Palermo, Italy. ${ }^{2}$ Department of Neonatology and NICU, University Hospital Policlinico P. Giaccone, Palermo, Italy. ${ }^{3}$ San Pietro Fatebenefratelli Hospital, UOSD Medical Genetics, Rome, Italy. ${ }^{4}$ Bambino Gesù Children's Hospital, Laboratory of Medical Genetics, Rome, Italy. 
Received: 23 January 2020 Accepted: 10 July 2020

Published online: 18 July 2020

\section{References}

1. Zhang M, Li FX, Liu XY, et al. TBX1 loss-of-function mutation contributes to congenital conotruncal defects. Exp Ther Med. 2018;15(1):447-53.

2. Volpe $P$, Paladini $D$, Marasini $M$, et al. Common arterial trunk in the fetus: characteristics, associations, and outcome in a multicentre series of 23 cases. Heart. 2003:89:1437-41.

3. Collett RW, Edwards JE. Persistent truncus arteriosus: a classification according to anatomic types. Surg Clin North Am. 1949;29:1245-70.

4. Yu S, Han J, Gao S, et al. The prenatal diagnosis of aortopulmonary window by fetal echocardiography. Echocardiography. 2018;35(11):1835-40.

5. Richardson JV, Doty DB, Rossi NP, et al. The spectrum of anomalies of aortopulmonary septation. J Thorac Cardiovasc Surg. 1979;78:21-7.

6. Ferencz C, Rubin JD, Loffredo CA, et al. Epidemiology of congenital heart disease. The Baltimore-Washington infant study. 1981-1989. Mount Kisco, New York: Futura Publishing Company Inc; 1993.

7. Chung I-M, Rajakumar G. Genetics of congenital heart defects: the NKX2-5 gene, a key player. Genes. 2016;7(2):6.

8. Nees SN, Chung WK. Genetic Basis of Human Congenital Heart Disease. Cold Spring Harb Perspect Biol. 2019;a036749. https://doi.org/10.1101/ cshperspect.a036749

9. Clark EB. Pathogenetic mechanism of congenital cardiovascular malformations revisited. Semin Perinatol. 1996;20(6):465-72.

10. Waldo KL, Hutson MR, Ward CC, et al. Secondary heart field contributes myocardium and smooth muscle to the arterial pole of the developing heart. Dev Biol. 2005;281:78-90.

11. Waldo K, Miyagawa-Tomita S, Kumiski D, et al. Cardiac neural crest cells provide new insight into septation of the cardiac outflow tract: aortic sac to ventricular septal closure. Dev Biol. 1998;196:129-44.

12. Marino B, Digilio MC. Congenital heart disease and genetic syndromes: specific correlation between cardiac phenotype and genotype. Cardiovasc Pathol. 2000;9(6):303-15.

13. Digilio MC, Silvestri LM, Dallapiccola B, et al. Le basi genetiche delle cardiopatie congenite. Cardiologia Pediatrica. 2014;175:173-86.

14. Wang $H$, Shen $\mathrm{Q}$, Ye LH, et al. MED12 mutations in human diseases. Protein Cell. 2013;4(9):643-6.

15. Hong SK, Haldin CE, Lawson ND, et al. The zebrafish kohtalo/trap230 gene is required for the development of the brain, neural crest, and pronephric kidney. Proc Natl Acad Sci U S A. 2005;102(51):18473-8.

16. Rau MJ, Fischer S, Neumann CJ. Zebrafish Trap230/Med12 is required as a coactivator for Sox9-dependent neural crest, cartilage and ear development. Dev Biol. 2006:296(1):83-93.

17. Segert J, Schneider I, Berger IM, et al. Mediator complex subunit Med12 regulates cardiac jelly development and AV valve formation in zebrafish. Prog Biophys Mol Biol. 2018;138:20-31.

18. Rocha PP, Scholze M, Bleiss W, et al. Med12 is essential for early mouse development and for canonical Wnt and Wnt/PCP signaling. Development. 2010:137(16):2723-31.

19. Schleiffarth JR, Person AD, Martinsen BJ. Wnt5a is required for cardiac outflow tract septation in mice. Pediatr Res. 2007;61(4):386-91.

\section{Publisher's Note}

Springer Nature remains neutral with regard to jurisdictional claims in published maps and institutional affiliations.

Ready to submit your research? Choose BMC and benefit from:

- fast, convenient online submission

- thorough peer review by experienced researchers in your field

- rapid publication on acceptance

- support for research data, including large and complex data types

- gold Open Access which fosters wider collaboration and increased citations

- maximum visibility for your research: over $100 \mathrm{M}$ website views per year

At BMC, research is always in progress.

Learn more biomedcentral.com/submissions 